Org Lett. 2015 July 02; 17(13): 3358-3361. doi:10.1021/acs.orglett.5b01629.

\title{
Total Synthesis of Oridamycins A and B
}

\author{
Adam H. Trotta* \\ Tri-Institutional PhD Program in Chemical Biology, Laboratory for Bioorganic Chemistry, Memorial \\ Sloan Kettering Cancer Center, Sloan Kettering Institute, 1275 York Avenue, New York, New York \\ 10065, United States
}

\begin{abstract}
The total synthesis of both oridamycin A and oridamycin B was accomplished starting from a common synthetic intermediate readily prepared from geranyl acetate. The sequence utilizes an oxidative radical cyclization to construct the trans-decalin ring system, setting three of four contiguous stereocenters in one operation. The carbazole nucleus was forged through a one-pot process entailing acid-promoted dehydration followed by $6 \pi$-electrocyclization/aromatization.
\end{abstract}

\section{Graphical abstract}

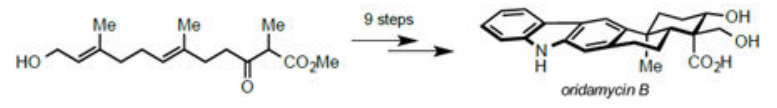

The xiamycin family of indolosesquiterpenes comprises bioactive compounds recently isolated from several strains of Streptomyces. ${ }^{1}$ Among the family members are xiamycin A (3), its biosynthetic precursor indosespene (4), and the homodimers dixiamycin $\mathrm{A} / \mathrm{B}(\mathbf{5})$, isolated as a mixture of atropisomers about the $N$-Nlinkage (Figure 1). These compounds display a range of interesting activities, including antiviral and antibacterial properties. ${ }^{1}$ Notably, the $N$-linked dimers dixiamycin A/B (5) are potent agents against both methicillin resistant Staphylococcus aureus 134/94 and vancomycin resistant Enterococcus faecalis 1528 R10. ${ }^{\text {le }}$ The closely related monomers oridamycin A (1) and oridamycin B (2) were isolated from Streptomyces sp. KS84 during screening for activity against Saprolegnia spp. - a water mold that infects freshwater fish, including those raised in commercial aquaculture. $^{2}$ These compounds have elicited significant interest within the synthetic community; efficient total syntheses of xiamycin A (3) and dixiamycin B (5) have been reported by Baran and coworkers, employing a cationic cyclization to construct the monomer and a novel electrochemical dimerization to generate the $N$-N linkage. ${ }^{3}$ Elegant syntheses of xiamycin A (3), indosespene (4), sespenine, oridamycin A (1), oridamycin B

\footnotetext{
*Corresponding Author ahtrotta@gmail.com.

Supporting Information

Experimental procedures and characterization data (including spectra for new compounds) is available free of charge via the Internet at http://pubs.acs.org.

Notes

The author declares no competing financial interests.
} 
(2), and dixiamycin $\mathrm{C}$ have been reported by the Li group, utilizing powerful radical cascades to construct the trans-decalin ring systems. ${ }^{4}$

Structurally, these pentacyclic molecules possess a carbazole nucleus fused to a trans-decalin ring system containing four contiguous stereocenters, including two quaternary centers. The major difference between the family members manifests at the C16 quaternary center; oridamycin A (1) and oridamycin B (2) each bear an axial carboxylic acid and an equatorial methyl/hydroxymethyl, while xiamycin A (3), indosespene (4), and dixiamycin A/B (5) contain an axially disposed methyl and an equatorial acid (Figure 1). Biosynthetically, it is known that xiamycin A (3) arises from precursor $\mathbf{6}$ through a selective enzymatic oxidation mediated by $X i a M$ (Figure 2, A) ${ }^{5}$ It seems plausible that intermediate 6 is also the biosynthetic precursor of the oridamycins, likely proceeding through an analogous methyl oxidation. Drawing inspiration from nature, we anticipated utilizing a common synthetic intermediate to access congeners from both the oridamycin and xiamycin families (Figure 2, B). Anticipating that biomimetic methyl oxidation of intermediate $\mathbf{6}$ would be difficult to replicate in the flask, we envisioned imparting the requisite oxidation pattern on a linear precursor that would be cyclized under two distinct sets of conditions to yield both of the desired C16 epimers. Employing an oxidative radical cyclization cascade under standard conditions would yield the stereochemistry associated with the oridamycins, proceeding through transition state $\mathbf{1 0}$ to produce intermediate $\mathbf{8}$ (Figure 2, C). ${ }^{6}$ To access the $\mathrm{C} 16$ stereoisomer relevant to the xiamycin family, we envisioned that introduction of a Lewis acid could invert the selectivity via chelation of the $\beta$-keto ester, producing transition state 11 en route to intermediate $\mathbf{9}^{7}$

We reasoned that it would be expeditious to initiate our synthetic efforts with the oridamycins, allowing for validation of late-stage transformations before attempting to invert the selectivity of the radical cyclization. Retrosynthetically, we anticipated that the C16 hydroxymethyl of oridamycin B (2) could arise from an oxime-directed, palladiumcatalyzed C-H oxidation of substrate 12 (Scheme 1). ${ }^{8}$ The C15 alcohol would arise via axial hydride delivery onto a suitable ketone precursor. The carbazole nucleus could be forged through $6 \pi$-electrocyclization/aromatization of an indole moiety appended to a diene system, which would itself arise from dehydration of a homo-allylic alcohol such as $\mathbf{1 3} .^{9}$ Grignard addition of a Boc-protected indole onto a suitable aldehyde would provide compound 13, containing all the requisite carbon atoms of the natural products. Finally, the trans-decalin ring system of $\mathbf{1 4}$ would be forged through an oxidative radical cyclization of a linear precursor readily derived from geranyl acetate. ${ }^{10}$ Oridamycin A (1) would be synthesized in analogous fashion without requiring the $\mathrm{C}-\mathrm{H}$ oxidation sequence.

In the forward direction, known compound $\mathbf{1 6}$ was readily obtained from geranyl acetate in multigram quantities in three operations (Scheme 2). ${ }^{10}$ Oxidative radical cyclization proceeded smoothly under the standard conditions to give alcohol 14 as a single diastereomer. ${ }^{11}$ Subsequent oxidation with Dess-Martin periodinane afforded the corresponding aldehyde, which was coupled with the Grignard reagent generated from 17 to produce $\mathbf{1 3}$ as an inconsequential mixture of diastereomers. ${ }^{9,12} \mathrm{Next}$, when considering the dehydration of intermediate $\mathbf{1 3}$, we hypothesized that the alcohol would be more prone to elimination if the Boc group were removed, allowing for the electron-rich indole to more 
readily participate in the process. To that end, substrate $\mathbf{1 3}$ was treated with TFA, and, to our surprise, the secondary alcohol was cleanly eliminated to form a triene intermediate with the Boc group still intact. Thus, this interesting result became the foundation of a one-pot process to forge the free carbazole directly from intermediate 13. In the event, treatment of a solution of 13 in $\mathrm{CH}_{2} \mathrm{Cl}_{2}$ with TFA formed the desired triene, which was not isolated but concentrated to remove solvent and acid. Next, this material was dissolved in toluene and heated to $135^{\circ} \mathrm{C}$ to induce a thermal $6 \pi$-electrocyclization/aromatization sequence under aerobic conditions. ${ }^{9}$ Upon completion, TFA was added at $45^{\circ} \mathrm{C}$ to remove the Boc group, yielding free carbazole 18. A stereoselective reduction using $\mathrm{NaBH}_{4}$ proceeded uneventfully, yielding oridamycin A methyl ester (d.r.> 20:1). The final remaining operation entailed conversion of the $\mathrm{C} 16$ axial methyl ester into the free acid. Initial attempts at saponification using various hydroxide sources were unproductive, likely due to the 1,3-diaxial relationship between the $\mathrm{C} 12$ methyl and the $\mathrm{C} 16$ ester-reminiscent of podocarpic acid ${ }^{13}$ — which may impede formation of the requisite tetrahedral intermediate. ${ }^{14}$ Accordingly, we utilized a nucleophilic cleavage protocol, allowing for successful dealkylation of the ester. Thus, upon treatment with $\mathrm{NaCN}$ in DMSO at elevated temperature, oridamycin A (1) was produced in $86 \%$ yield, cleanly affording characterization quality material. ${ }^{15}$

To access oridamycin B (2), the route to oridamycin A (1) was diverted from intermediate 13. The same sequence of dehydration and electrocyclization was employed, but the Boc group was preserved to produce the protected carbazole. The ketone was smoothly converted into $O$-methyloxime derivative $\mathbf{1 2}$ as a prerequisite for the selective, late-stage $\mathrm{C}-\mathrm{H}$ oxidation. Upon treatment with $\mathrm{PhI}(\mathrm{OAc})_{2}$ and catalytic $\mathrm{Pd}(\mathrm{OAc})_{2}$ at elevated temperature, compound 19 was cleanly produced in 69\% yield. Next, global deprotection using a 3:1 mixture of aq $1 \mathrm{M} \mathrm{HCl}$ :acetone at $80{ }^{\circ} \mathrm{C}$ cleaved the acetate, removed the Boc group, and hydrolyzed the $O$-methyloxime. A variety of conditions were screened for this transformation, most of which produced a significant amount of a presumed retro-Aldol product, which was largely suppressed through the use of acetone as a cosolvent. However, the elevated temperature required to cleave the $O$-methyloxime appeared to cause a significant amount of nonspecific decomposition, resulting in diminished recovery. The product of the global deprotection was unstable to silica, requiring reduction of the crude material to produce oridamycin B methyl ester. Finally, treatment of this compound with $\mathrm{NaCN}$ produced oridamycin B (2). Interestingly, it was found that the ${ }^{1} \mathrm{H}$ NMR spectrum of 2 was $\mathrm{pH}$ dependent (see Supporting Information).

With the successful synthesis of the oridamycins, the next phase of our program is aimed at accessing epimeric intermediate 9 via Lewis acid chelation of the radical intermediate during cyclization (see Figure 2). ${ }^{7}$ Realization of this strategy could provide access to the xiamycin family from the same linear precursor 7 used to generate the oridamycins. Furthermore, we are optimistic that this transformation could be rendered asymmetric through the use of a suitable chiral ligand. ${ }^{16}$ Efforts are ongoing in our laboratory to validate these assertions.

In conclusion, both oridamycin A and B were synthesized from known compound $\mathbf{1 6}$ in six and nine steps, respectively. The synthesis was diverted from common intermediate $\mathbf{1 3}$ harboring all of the carbon atoms contained within each natural product. The successful 
construction of the oridamycins lays the foundation for a unified synthetic strategy for this entire class of indolosesquiterpenes.

\section{Supplementary Material}

Refer to Web version on PubMed Central for supplementary material.

\section{Acknowledgments}

Prof. Samuel J. Danishefsky (MSKCC) is foremost acknowledged for his intellectual mentorship. This material is based upon work supported by the National Science Foundation Graduate Research Fellowship under Grant No. 2014157713. The MSKCC core facility is also acknowledged for mass spectrometric assistance and for providing the necessary instrumentation.

\section{References}

1. (a) Ding L, Münch J, Goerls H, Maier A, Fiebig HH, Lin WH, Hertweck C. Bioorg Med Chem Lett. 2010; 20:6685-6687. [PubMed: 20880706] (b) Ding L, Maier A, Fiebig HH, Lin WH, Hertweck C. Org Biomol Chem. 2011; 9:4029-4031. [PubMed: 21528153] (c) Zhang Q, Mandi A, Li SM, Chen Y, Zhang W, Tian XP, Zhang H, Li HX, Zhang W, Zhang S, Ju JH, Kurtan T, Zhang C. Eur J Org Chem. 2012:5256-5262.(d) Xu ZL, Baunach M, Ding L, Hertweck C. Angew Chem Int Ed. 2012; 51:10293-10297.(e) Baunach M, Ding L, Bruhn T, Bringmann G, Hertweck C. Angew Chem Int Ed. 2013; 52:9040-9043.

2. Takada K, Kajiwara H, Imamura N. J Nat Prod. 2010; 73:698-701. [PubMed: 20369841]

3. Rosen BR, Werner EW, O’Brien AG, Baran PS. J Am Chem Soc. 2014; 136:5571-5574. [PubMed: 24697810]

4. Meng Z, Yu H, Li L, Tao W, Chen H, Wan M, Yang P, Edmonds DJ, Zhong J, Li A. Nat Commun. 2015; :6.doi: 10.1038/ncomms7096. NOTE: This closely related publication appeared just as our own studies were being completed. Sun Y, Chen PX, Zhang DL, Baunach M, Hertweck C, Li A. Angew Chem Int Ed. 2014; 53:9012-9016.

5. Zhang Q, Li H, Li S, Zhu Y, Zhang G, Zhang H, Zhang W, Shi R, Zhang C. Org Lett. 2012; 14:6142-6145. [PubMed: 23205935]

6. Snider BB. Chem Rev. 1996; 96:339-363. [PubMed: 11848756] . and references therein.

7. Yang D, Ye XY, Xu M, Pang KW, Cheung KK. J Am Chem Soc. 2000; 122:1658-1663.

8. Desai LV, Hull KL, Sanford MS. J Am Chem Soc. 2004; 126:9542-9543. [PubMed: 15291549]

9. Bian M, Wang Z, Xiong X, Sun Y, Matera C, Nicolaou KC, Li A. J Am Chem Soc. 2012; 134:80788081. [PubMed: 22537293]

10. (a) González MA, Molina-Navarro S. J Org Chem. 2007; 72:7462-7465. [PubMed: 17713953] (b) Jenny L, Borschberg HJ. Helv Chim Acta. 1995; 78:715-731.(c) Kitagawa Y, Itoh A, Hashimoto S, Yamamoto H, Nozaki H. J Am Chem Soc. 1977; 99:3864-3867.

11. Yang D, Xu M. Org Lett. 2001; 3:1785-1788. [PubMed: 11405711]

12. Leung JC, Patman RL, Sam B, Krische MJ. Chem Eur J. 2011; 17:12437-12443. [PubMed: 21953608]

13. (a) Wenkert E, Afonso A, Bredenberg JBs, Kaneko C, Tahara A. J Am Chem Soc. 1964; 86:20382043.(b) Wenkert E, Jackson BG. J Am Chem Soc. 1958; 80:217-219.(c) Sherwood IR, Short WF. J Chem Soc (Resumed). 1938:1006-1013.

14. Reddy BVS, Reddy LR, Corey EJ. Tetrahedron Lett. 2005; 46:4589-4593.

15. O’Brien EM, Morgan BJ, Kozlowski MC. Angew Chem Int Ed. 2008; 47:6877-6880.

16. (a) Yang D, Zheng BF, Gao Q, Gu S, Zhu NY. Angew Chem Int Ed. 2006; 45:255-258.(b) Sibi MP, Ji JG, Wu JH, Gurtler S, Porter NA. J Am Chem Soc. 1996; 118:9200-9201. 


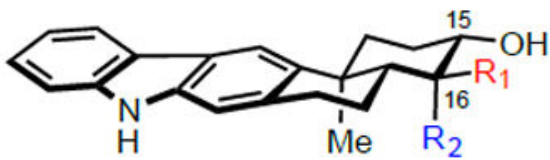

1, oridamycin $A: \mathrm{R}_{1}=\mathrm{Me}$

$\mathrm{H}$

Me $\mathrm{R}_{2}$

$\mathrm{R}_{2}=\mathrm{CO}_{2} \mathrm{H}$

2, oridamycin B: $\mathrm{R}_{1}=\mathrm{CH}_{2} \mathrm{OH}$

$\mathrm{R}_{2}=\mathrm{CO}_{2} \mathrm{H}$

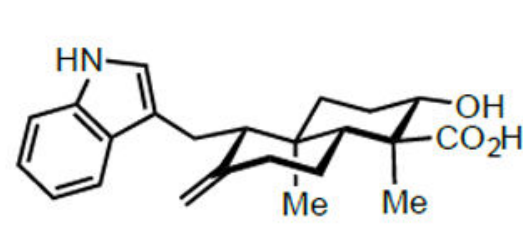

3, xiamycin $\mathrm{A}: \quad \mathrm{R}_{1}=\mathrm{CO}_{2} \mathrm{H}$

$\mathrm{R}_{2}=\mathrm{Me}$

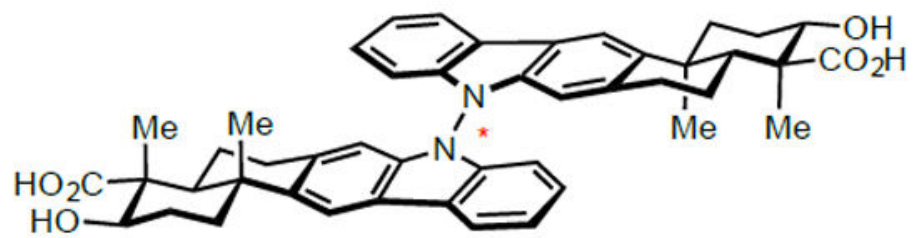

5, dixiamycin $A$ \& $B$

Figure 1.

Selected members of the oridamycin and xiamycin families. 
A.

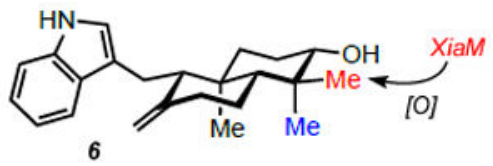

B.

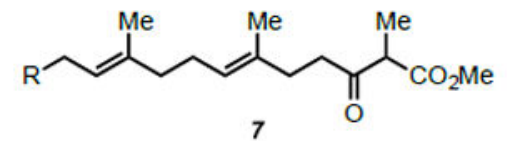

'standard cyclization

7 conditions'

Lewis acid

catalyzed
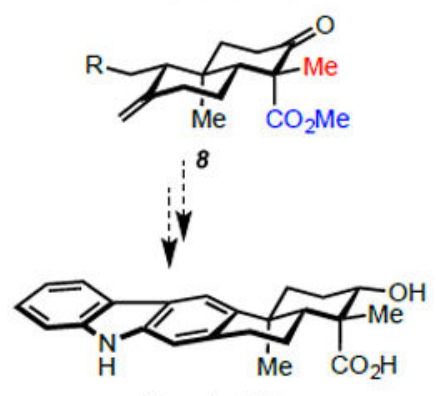

oridamycin A, 1

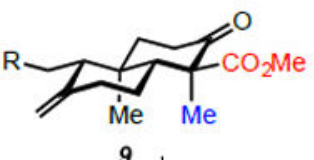

9

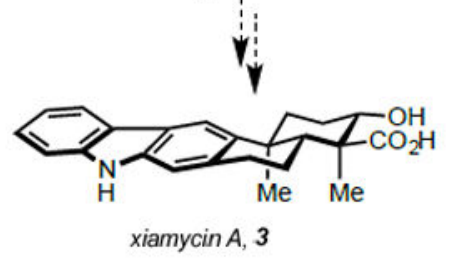

C.

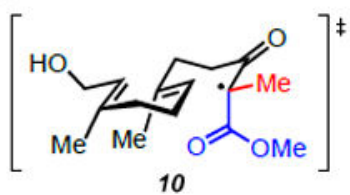

vs.

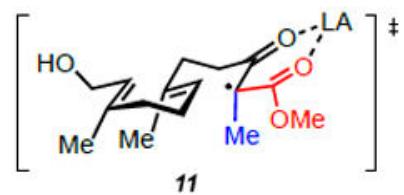

Figure 2.

A. Biosynthetic precursor for the xiamycin family, and proposed precursor for the oridamycin family, B. General strategy to access both the xiamycin and oridamycin families from a common linear precursor, C. Proposed radical transition states for the cyclization reactions. 


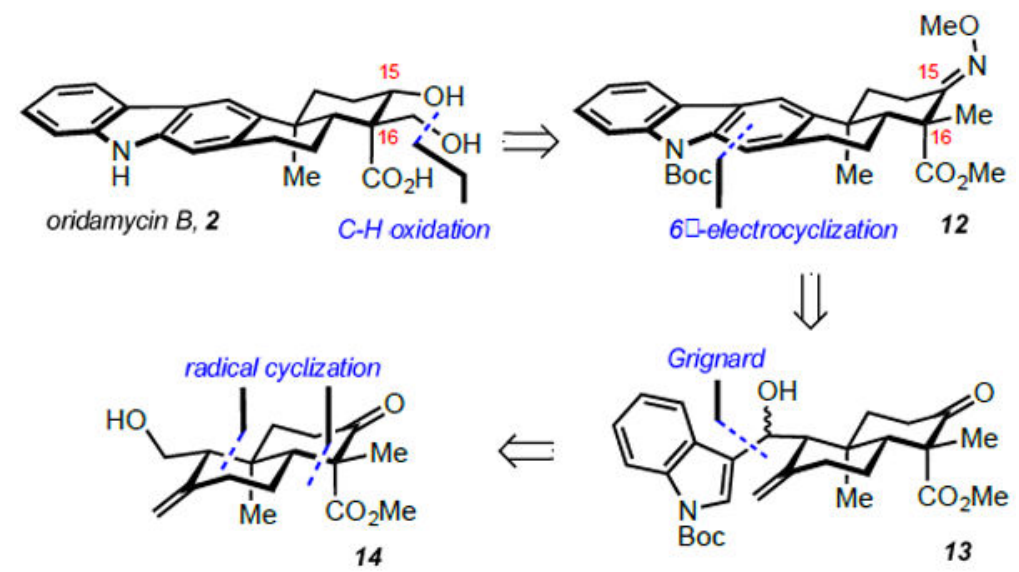

Scheme 1.

Retrosynthesis of Oridamycin B 


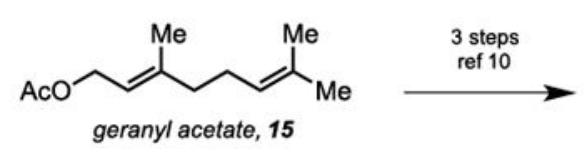

geranyl acetate, 15

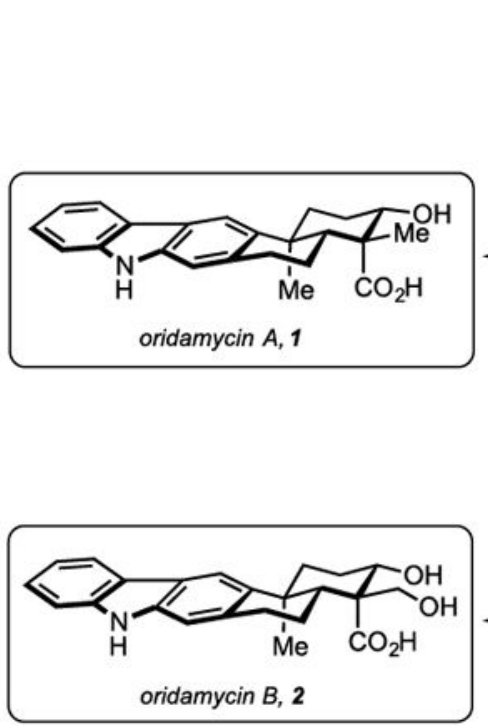

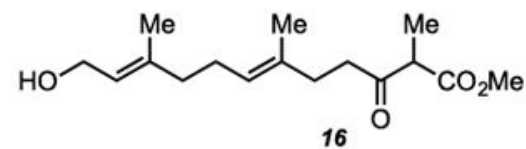

16

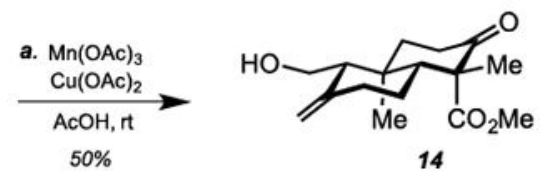
Dess-Martin periodinane $\mathrm{CH}_{2} \mathrm{Cl}_{2}, 0^{\circ} \mathrm{C}$

$73 \%$

c. $17, \mathrm{EtMgBr}$ THF, $0^{\circ} \mathrm{C}$

† $99 \%$

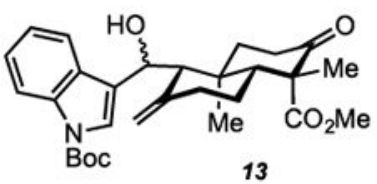

18

g. TFA, $\mathrm{CH}_{2} \mathrm{Cl}_{2}$; $\mathrm{PhMe}, 140^{\circ} \mathrm{C}$

$68 \%$

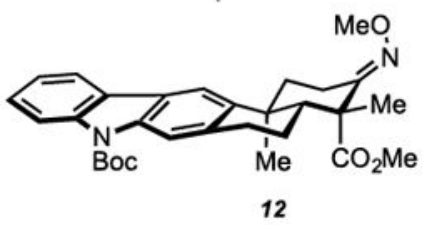

Scheme 2.

Synthesis of Oridamycin A and B 